\title{
Improved network analysis of coupled antenna diversity performance
}

Michael A. Jensen

jensen@byu.edu

Matthew L. Morris

Follow this and additional works at: https://scholarsarchive.byu.edu/facpub

Part of the Electrical and Computer Engineering Commons

\section{Original Publication Citation}

Morris, M. L., and M. A. Jensen. "Improved Network Analysis of Coupled Antenna Diversity Performance." Wireless Communications, IEEE Transactions on 4.4 (25): 1928-34

\section{BYU ScholarsArchive Citation}

Jensen, Michael A. and Morris, Matthew L., "Improved network analysis of coupled antenna diversity performance" (2005). Faculty Publications. 362.

https://scholarsarchive.byu.edu/facpub/362 


\title{
Improved Network Analysis of Coupled Antenna Diversity Performance
}

\author{
Matthew L. Morris and Michael A. Jensen, Senior Member, IEEE
}

\begin{abstract}
This paper presents a new framework for the analysis of mutually coupled diversity antennas based on network theory. The network model incorporates the matching network between the antennas and front-end amplifiers and uses a realistic model for the amplifier noise. The resulting analysis includes the impact of the coupled-antenna radiation patterns and impedance characteristics in determining the branch signal-to-noise ratios (SNRs). Application of the formulation to coupled dipole antennas characterized using full-wave electromagnetic analysis illustrates that a matching network providing a minimum amplifier noise figure achieves significantly better diversity performance than one providing maximum power transfer.
\end{abstract}

Index Terms-Antenna diversity, impedance matching, mutual coupling, noise figure.

\section{INTRODUCTION}

A NTENNA diversity has long been an important technique for mitigating the detrimental effects of multipath fading in wireless communication links [1]. This technology is likely to become even more prevalent in future systems as the demand for spectral efficiency increases. However, when multiple antennas are placed on small personal communications devices, the close element spacing leads to mutual coupling that alters the antenna terminal impedance and radiation pattern [2], thereby impacting the diversity performance.

Past work on coupled-antenna diversity performance has emphasized the effect of coupling on the radiation pattern and resulting signal correlation and has either ignored the impact of the altered antenna impedance [3]-[9] or given only limited consideration to the receive impedance matching problem [10]-[15]. In prior work, a network model that includes the effect of pattern and impedance on the received signal voltage was developed [16]. However, the simple model for the front-end noise did not accurately represent noise generated in realistic amplifiers. The analysis, therefore, confirmed prior findings that matching for maximum power transfer is optimal for maximizing diversity performance [11], [12].

In this paper, the network analysis of coupled-antenna diversity receivers is extended to include an improved amplifier noise model. This model, while creating significantly increased analysis complexity, allows proper characterization of antenna

Manuscript received October 30, 2003; revised April 16, 2004; accepted June 4, 2004. The editor coordinating the review of this paper and approving it for publication is R. Murch. This work was supported by the National Science Foundation under Information Technology Research Grants CCR-0081476 and CCR-0313056.

The authors are with the Department of Electrical and Computer Engineering, Brigham Young University, Provo, UT 84602 USA.

Digital Object Identifier 10.1109/TWC.2005.852135

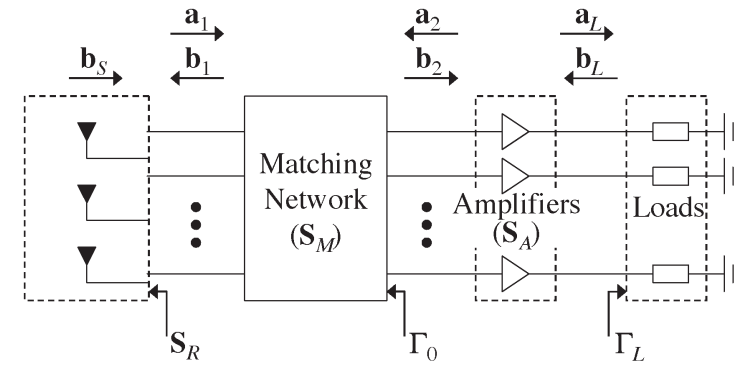

Fig. 1. Block diagram of the diversity receiver including mutually coupled array, matching network, receiver amplifiers, and loads.

diversity architectures due to its realistic representation of signal-to-noise ratio (SNR). In fact, examples using the analysis framework with electromagnetically characterized coupled dipoles reveal that matching for a minimum amplifier noise figure can be far superior to matching for optimal power transfer (50\% improvement for the transistor used).

Section II provides a characterization of the blocks forming the diversity receiver architecture and introduces the noisy amplifier model. Sections III and IV then detail the network analysis used to obtain the voltage signals at the amplifier outputs for a given incident field and apply this framework to quantify the diversity performance of the coupled antenna system. Computational results showing the performance of two coupled dipoles as a function of antenna spacing for a representative transistor are given in Section V. Finally, concluding remarks are given in Section VI.

\section{Diversity Receiver Model}

Careful characterization of an antenna diversity receiving system requires the construction of a detailed model that includes the multipath propagation channel, coupled antenna, matching network, and receiving amplifiers with appropriate load terminations. Fig. 1 shows a block diagram of this system (without the propagation model, which is discussed in Section IV-A). For analysis purposes, a scattering parameter (S-parameter) [17] description of the network signals is adopted wherein the forward and reverse traveling waves are denoted as $\mathbf{a}$ and $\mathbf{b}$, respectively. All S-parameters are referenced to a real impedance $Z_{0}$. The flow diagram for this network, with the various blocks delineated by the dashed lines, appears in Fig. 2. Each of the blocks will be described in the following sections. Throughout this analysis, boldface uppercase and lowercase letters will describe matrices and column vectors, respectively, with $H_{m n}$ denoting the element occupying the $m$ th row and $n$th 


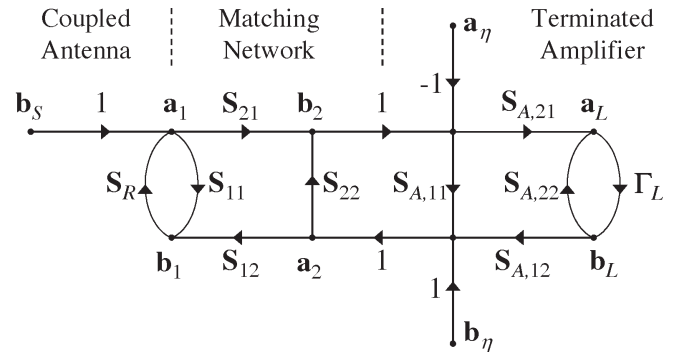

Fig. 2. Flow diagram representation of the diversity receiver depicted in Fig. 1.

column of the matrix $\mathbf{H}$, and $h_{m}$ representing the $m$ th element of the vector $h$.

\section{A. Coupled Antenna}

Within the context of antenna diversity systems, mutual coupling alters the performance from the isolated antenna element case in two fashions. First, the introduction of elements in the vicinity of a radiating antenna changes the electromagnetic boundary conditions and, therefore, alters the driven-element radiation pattern. Second, excitation of one element induces a voltage across the terminals of adjacent elements: an effect represented here for the $N$ antennas by a terminal $N \times N$ $S$-matrix $\mathbf{S}_{R}$. A combination of these two factors will influence the $N \times 1$ vector of source signals $\mathbf{b}_{S}$, which results when an electromagnetic field is incident on the array and the antenna ports are terminated in the reference impedance $Z_{0}$. With this description, the total signal entering the network from the antenna terminals can be expressed as

$$
\mathbf{a}_{1}=\mathbf{b}_{S}+\mathbf{S}_{R} \mathbf{b}_{1}
$$

A mechanism for computing $\mathbf{b}_{S}$ is discussed in Section III-A.

\section{B. Matching Network}

The matching network in Fig. 1 provides a proper impedance interface between the antenna and receiving amplifiers. Given the multiport nature of the diversity receiving system, the matching network will be represented using a block matrix S-parameter description, or

$$
\mathbf{S}_{M}=\left[\begin{array}{ll}
\mathbf{S}_{11} & \mathbf{S}_{12} \\
\mathbf{S}_{21} & \mathbf{S}_{22}
\end{array}\right]
$$

where 1 and 2 refer to input and output ports, respectively.

Incorporating the coupled nature of the antenna when synthesizing an optimal matching network is a relatively complex undertaking. The goal here is to determine the mathematical form of the S-matrix of such a matching network based on design goals. To facilitate this specification, the present discussion will be restricted to lossless matching networks that ideally have unity noise figures and are characterized by unitary S-matrices. Note that this constraint generally precludes the use of resistive or active matching network elements that add noise. As detailed in the Appendix, the singular value decomposition (SVD) of the subblocks $\mathbf{S}_{i j}=\mathbf{U}_{i j} \Lambda_{i j}^{1 / 2} \mathbf{V}_{i j}^{\mathrm{H}}$ in (2) can be taken, where $\mathbf{U}_{i j}$ and $\mathbf{V}_{i j}$ are unitary matrices of singular vectors, $\Lambda_{i j}^{1 / 2}$ is a diagonal matrix of real singular values, and $\{\cdot\}^{H}$ represents a conjugate transpose. Since $\mathbf{S}_{M}$ is unitary, relationships exist among the subblock singular vectors and values, leading to the forms

$$
\begin{aligned}
& \mathbf{S}_{11}=\mathbf{U}_{11} \boldsymbol{\Lambda}_{11}^{\frac{1}{2}} \mathbf{V}_{11}^{\mathrm{H}} \\
& \mathbf{S}_{12}=-\mathbf{U}_{11} \boldsymbol{\Theta}^{\mathrm{H}}\left(\mathbf{I}-\boldsymbol{\Lambda}_{11}\right)^{\frac{1}{2}} \mathbf{V}_{22}^{\mathrm{H}} \\
& \mathbf{S}_{21}=\mathbf{U}_{22} \boldsymbol{\Theta}\left(\mathbf{I}-\boldsymbol{\Lambda}_{11}\right)^{\frac{1}{2}} \mathbf{V}_{11}^{\mathrm{H}} \\
& \mathbf{S}_{22}=\mathbf{U}_{22} \boldsymbol{\Lambda}_{11}^{\frac{1}{2}} \mathbf{V}_{22}^{\mathrm{H}}
\end{aligned}
$$

where $\mathbf{I}$ is the identity matrix, and $\Theta$ is a diagonal phase shift matrix with arbitrary complex elements of unit magnitude. These relations form a key part of the matching network specification in Section III-C.

It is interesting to consider the physical implication of the SVD representation of $\mathbf{S}_{i j}$. Since $\mathbf{b}_{i}=\mathbf{S}_{i j} \mathbf{a}_{j}$ for $\mathbf{a}_{k}=\mathbf{0}, k \neq j$

$$
\mathbf{b}_{i}^{\prime}=\mathbf{U}_{i j}^{\mathrm{H}} \mathbf{b}_{i}=\Lambda_{i j}^{\frac{1}{2}} \mathbf{V}_{i j}^{\mathrm{H}} \mathbf{a}_{j}=\boldsymbol{\Lambda}_{i j}^{\frac{1}{2}} \mathbf{a}_{j}^{\prime} .
$$

Considering vectors $\mathbf{b}_{i}^{\prime}$ and $\mathbf{a}_{j}^{\prime}$, the response of the network is now characterized by $N$ one-port elements, where the $n$th element presents a positive reflection or transmission coefficient $\Lambda_{i j, n n}^{1 / 2}$.

\section{Noisy Amplifiers}

The complication considered in this paper is the noise model associated with practical high-frequency transistor-based amplifiers [18]. A power wave noise model is adopted [19], wherein the $m$ th amplifier is described by forward and reverse traveling noise waves $a_{\eta, m}$ and $b_{\eta, m}$, respectively, at the amplifier input. Using the notation of Fig. 2 with the amplifier S-parameters denoted with a subscript " $A$," the signal plus noise amplifier output waves are

$$
\begin{aligned}
\mathbf{a}_{2} & =\mathbf{S}_{A, 11} \mathbf{b}_{2}+\mathbf{S}_{A, 12} \mathbf{b}_{L}-\mathbf{S}_{A, 11} \mathbf{a}_{\eta}+\mathbf{b}_{\eta} \\
\mathbf{a}_{L} & =\mathbf{S}_{A, 21} \mathbf{b}_{2}+\mathbf{S}_{A, 22} \mathbf{b}_{L}-\mathbf{S}_{A, 21} \mathbf{a}_{\eta} .
\end{aligned}
$$

If it is assumed that the noise in each amplifier is uncorrelated with that of all other amplifiers, then

$$
\begin{aligned}
& E\left\{\mathbf{a}_{\eta} \mathbf{a}_{\eta}^{\mathrm{H}}\right\}=k_{\mathrm{B}} T_{\alpha} B \mathbf{I} \\
& E\left\{\mathbf{b}_{\eta} \mathbf{b}_{\eta}^{\mathrm{H}}\right\}=k_{\mathrm{B}} T_{\beta} B \mathbf{I} \\
& E\left\{\mathbf{a}_{\eta} \mathbf{b}_{\eta}^{\mathrm{H}}\right\}=k_{\mathrm{B}} T_{\gamma}^{*} B \mathbf{I}
\end{aligned}
$$

where $k_{\mathrm{B}}$ is the Boltzmann constant, $B$ is the system noise power bandwidth, $\mathrm{E}\{\cdot\}$ denotes an expectation, and $T_{\alpha}, T_{\beta}$, and $T_{\gamma}$ are effective noise temperatures [19].

\section{Network ANALYSIS}

The goal of the network analysis is to determine the received signal and noise at the amplifier output for a given incident electric field distribution. The formulation will allow us to 
specify the matching network blocks $\mathbf{S}_{i j}$ to achieve design goals.

\section{A. Antenna Port Output Signal}

First, the signal vector $\mathbf{b}_{S}$ at the receive antenna ports must be determined for an incident plane wave. Let $\mathbf{f}_{n}(\Omega)$ represent the vector far-zone electric field of the $n$th receive element for unit driving current $\left(i_{n}=1\right)$ with all other elements open circuited $\left(i_{k}=0\right.$ for $k \neq n$ ), normalized by the field of an isotropic radiator, where $\Omega$ is the solid angle direction. The total normalized (dimensionless) field pattern at the angle $\Omega$ for the array is then expressed using the superposition

$$
\mathbf{e}(\Omega)=\sum_{n} \mathbf{f}_{n}(\Omega) i_{n}=\mathbf{F}(\Omega) \mathbf{i}
$$

where $\mathbf{i}$ is the column vector of excitation currents, and $\mathbf{f}_{n}(\Omega)$ represents the $n$th column of the matrix $\mathbf{F}(\Omega)$.

Now, assume that a plane wave arrives from the direction $\Omega_{0}$ with complex field strength $E_{0}$ and electric field polarization vector $\hat{e}$. The open-circuit antenna voltages are proportional to the inner product between the vector field intensity and the vector antenna response in the direction $\Omega_{0}$, or [11]

$$
\mathbf{v}=2 c_{1} E_{0} \mathbf{F}\left(\Omega_{0}\right)^{T} \hat{e}
$$

where $c_{1}$ is a complex constant (in ampere meters), and the factor of 2 is for later convenience. Furthermore, if the antenna ports are open circuited in the model in Fig. 1, the situation that $\mathbf{b}_{1}=\mathbf{a}_{1}$ holds, so that this voltage can also be expressed as

$$
\mathbf{v}=Z_{0}^{\frac{1}{2}}\left(\mathbf{a}_{1}+\mathbf{b}_{1}\right)=2 Z_{0}^{\frac{1}{2}} \mathbf{a}_{1}
$$

Using $\mathbf{b}_{1}=\mathbf{a}_{1}$ in (1) leads to $\mathbf{b}_{S}=\left(\mathbf{I}-\mathbf{S}_{R}\right) \mathbf{a}_{1}$, which, when used with (9) and (10), gives the result

$$
\begin{aligned}
\mathbf{b}_{S} & =c_{1} Z_{0}^{-\frac{1}{2}} E_{0}\left(\mathbf{I}-\mathbf{S}_{R}\right) \mathbf{F}\left(\Omega_{0}\right)^{T} \hat{e} \\
& =c_{1} Z_{0}^{-\frac{1}{2}} E_{0} \mathbf{F}^{\prime}\left(\Omega_{0}\right)^{T} \hat{e}
\end{aligned}
$$

where the impedance mismatch factor $\left(\mathbf{I}-\mathbf{S}_{R}\right)$ has been included in the effective pattern $\mathbf{F}^{\prime}\left(\Omega_{0}\right)$ for simplicity.

\section{B. Amplifier Output Signal}

Given a value of $\mathbf{b}_{S}$ for an incident field and receive array, the received signal plus noise delivered to the loads at the amplifier outputs can now be determined. To begin, (1) is used with $\mathbf{b}_{1}=$ $\mathbf{S}_{11} \mathbf{a}_{1}+\mathbf{S}_{12} \mathbf{a}_{2}$ to obtain

$$
\mathbf{a}_{1}=\left(\mathbf{I}-\mathbf{S}_{R} \mathbf{S}_{11}\right)^{-1}\left(\mathbf{b}_{S}+\mathbf{S}_{R} \mathbf{S}_{12} \mathbf{a}_{2}\right) .
$$

Using (12) with $\mathbf{b}_{2}=\mathbf{S}_{21} \mathbf{a}_{1}+\mathbf{S}_{22} \mathbf{a}_{2}$, leads to:

$$
\begin{aligned}
\mathbf{b}_{2}=\mathbf{S}_{21}\left(\mathbf{I}-\mathbf{S}_{R} \mathbf{S}_{11}\right)^{-1} \mathbf{b}_{S} \\
+\underbrace{\left[\mathbf{S}_{22}+\mathbf{S}_{21}\left(\mathbf{I}-\mathbf{S}_{R} \mathbf{S}_{11}\right)^{-1} \mathbf{S}_{R} \mathbf{S}_{12}\right]}_{\boldsymbol{\Gamma}_{0}} \mathbf{a}_{2}
\end{aligned}
$$

where $\Gamma_{0}$ has been used to represent the reflection coefficient at the matching network output (see Fig. 1).

Now, according to Fig. 2, $\mathbf{a}_{2}$ can be expressed as

$$
\mathbf{a}_{2}=\mathbf{b}_{\eta}+\mathbf{S}_{A, 11}\left(\mathbf{b}_{2}-\mathbf{a}_{\eta}\right)+\mathbf{S}_{A, 12} \boldsymbol{\Gamma}_{L} \mathbf{a}_{L}
$$

Inserting this result into (13) and rearranging leads to

$$
\begin{aligned}
\mathbf{b}_{2}=\left(\mathbf{I}-\boldsymbol{\Gamma}_{0} \mathbf{S}_{A, 11}\right)^{-1}\left[\mathbf{S}_{21}\left(\mathbf{I}-\mathbf{S}_{R} \mathbf{S}_{11}\right)^{-1} \mathbf{b}_{S}\right. \\
\left.+\boldsymbol{\Gamma}_{0}\left(\mathbf{b}_{\eta}-\mathbf{S}_{A, 11} \mathbf{a}_{\eta}+\mathbf{S}_{A, 12} \boldsymbol{\Gamma}_{L} \mathbf{a}_{L}\right)\right]
\end{aligned}
$$

Rearranging the expression $\mathbf{a}_{L}=\mathbf{S}_{A, 21}\left(\mathbf{b}_{2}-\mathbf{a}_{\eta}\right)+$ $\mathbf{S}_{A, 22} \boldsymbol{\Gamma}_{L} \mathbf{a}_{L}$, produces

$$
\mathbf{a}_{L}=\left(\mathbf{I}-\mathbf{S}_{A, 22} \boldsymbol{\Gamma}_{L}\right)^{-1} \mathbf{S}_{A, 21}\left(\mathbf{b}_{2}-\mathbf{a}_{\eta}\right)
$$

Substituting (15) into (16), simplifying, and subsequently using the fact that the voltage across the load termination is $\mathbf{v}_{L}=$ $Z_{0}^{1 / 2}\left(\mathbf{I}+\boldsymbol{\Gamma}_{L}\right) \mathbf{a}_{L}$ leads to a final expression

$$
\mathbf{v}_{L}=\mathbf{Q}[\underbrace{\mathbf{S}_{21}\left(\mathbf{I}-\mathbf{S}_{R} \mathbf{S}_{11}\right)^{-1}}_{\mathbf{G}} \mathbf{b}_{S}+\boldsymbol{\Gamma}_{0} \mathbf{b}_{\eta}-\mathbf{a}_{\eta}]
$$

where

$$
\begin{aligned}
\mathbf{Q}=Z_{0}^{\frac{1}{2}}\left(\mathbf{I}+\boldsymbol{\Gamma}_{L}\right) & {\left[\left(\mathbf{I}-\boldsymbol{\Gamma}_{0} \mathbf{S}_{A, 11}\right) \mathbf{S}_{A, 21}^{-1}\right.} \\
& \left.\times\left(\mathbf{I}-\mathbf{S}_{A, 22} \boldsymbol{\Gamma}_{L}\right)-\boldsymbol{\Gamma}_{0} \mathbf{S}_{A, 12} \boldsymbol{\Gamma}_{L}\right]^{-1} .
\end{aligned}
$$

\section{Matching Network Specification}

Practical amplifier design involves specifying a design goal for the amplifier performance (minimum noise figure, maximum power transfer) and synthesizing the source and load terminations that achieve this goal [18]. In this analysis, this is tantamount to defining a desired value of $\boldsymbol{\Gamma}_{0}$, which is the source termination seen by the amplifier, and using this value to determine the subblocks $\mathbf{S}_{i j}$.

Assuming that $\boldsymbol{\Gamma}_{0}$ has been given, the form for $\boldsymbol{\Gamma}_{0}$ in (13) coupled with the expressions in (3) is used to obtain

$$
\begin{aligned}
\boldsymbol{\Gamma}_{0} & =\mathbf{U}_{0} \boldsymbol{\Lambda}_{0}^{\frac{1}{2}} \mathbf{V}_{0}^{\mathrm{H}} \\
& =\mathbf{U}_{22}\left[\boldsymbol{\Lambda}_{11}^{\frac{1}{2}}-\left(\mathbf{I}-\boldsymbol{\Lambda}_{11}\right)^{\frac{1}{2}} \mathbf{T}\left(\mathbf{I}-\boldsymbol{\Lambda}_{11}\right)^{\frac{1}{2}}\right] \mathbf{V}_{22}^{\mathrm{H}} \\
\mathbf{T} & =\boldsymbol{\Theta} \mathbf{V}_{11}^{\mathrm{H}}\left(\mathbf{I}-\mathbf{S}_{R} \mathbf{U}_{11} \boldsymbol{\Lambda}_{11}^{\frac{1}{2}} \mathbf{V}_{11}^{\mathrm{H}}\right)^{-1} \mathbf{S}_{R} \mathbf{U}_{11} \boldsymbol{\Theta}^{\mathrm{H}}
\end{aligned}
$$

where the first equality is the SVD of $\boldsymbol{\Gamma}_{0}$. There is flexibility in specifying the singular vectors $\mathbf{U}_{i i}$ and $\mathbf{V}_{i i}, i \in 1,2$, and therefore, representations that lead to mathematical simplicity are chosen. First, it is clear that if $\mathbf{S}_{R}=\mathbf{U}_{R} \boldsymbol{\Lambda}_{R}^{1 / 2} \mathbf{V}_{R}^{\mathrm{H}}$, then by choosing $\mathbf{U}_{11}=\mathbf{V}_{R}$ and $\mathbf{V}_{11}=\mathbf{U}_{R}$, leads to the expression

$$
\mathbf{T}=\left(\mathbf{I}-\boldsymbol{\Lambda}_{R}^{\frac{1}{2}} \boldsymbol{\Lambda}_{11}^{\frac{1}{2}}\right)^{-1} \boldsymbol{\Lambda}_{R}^{\frac{1}{2}}
$$


which is diagonal. If $\mathbf{U}_{22}=\mathbf{U}_{0}$ and $\mathbf{V}_{22}=\mathbf{V}_{0}$ are further chosen, (19) can be solved to obtain

$$
\boldsymbol{\Lambda}_{11}^{\frac{1}{2}}=\left(\boldsymbol{\Lambda}_{0}^{\frac{1}{2}}+\boldsymbol{\Lambda}_{R}^{\frac{1}{2}}\right)\left(\mathbf{I}+\boldsymbol{\Lambda}_{0}^{\frac{1}{2}} \Lambda_{R}^{\frac{1}{2}}\right)^{-1} .
$$

The matrix $\Theta$ can be any diagonal matrix with entries of unit magnitude, and therefore, $\boldsymbol{\Theta}=j \mathbf{I}$ is used.

If the amplifiers are assumed uncoupled $\left(\mathbf{S}_{A, i j}\right.$ and $\boldsymbol{\Gamma}_{L}$ are diagonal), typical design goals will be achieved for diagonal $\Gamma_{0}$. Let $\Gamma_{\mathrm{opt}}$ and $\Gamma_{\mathrm{MS}}$ represent the (scalar) source reflection coefficient for achieving minimum noise figure and maximum power gain, respectively, for the amplifier. These values are readily computed from the specifications of the amplifying device [18]. Then, achieving minimum noise figure and optimal power gain are accomplished by setting $\boldsymbol{\Gamma}_{0}=\Gamma_{\mathrm{opt}} \mathbf{I}$ and $\boldsymbol{\Gamma}_{0}=$ $\Gamma_{\mathrm{MS}} \mathbf{I}$, respectively. Since diversity performance depends on SNR, a design for minimum noise figure is expected to outperform one for optimal power gain. It is also pointed out that if $\boldsymbol{\Gamma}_{0}=\mathbf{0}$, then any reverse traveling noise from the transistor will not be reflected back into the amplifier, leading to perhaps reduced SNR compared to a design for optimal power gain. This condition can be obtained by setting $\mathbf{S}_{11}=\mathbf{S}_{R}^{\mathrm{H}}$, which is easily verified by substituting $\mathbf{S}_{R}=\mathbf{S}_{11}^{\mathrm{H}}=\mathbf{V}_{11} \boldsymbol{\Lambda}_{11}^{1 / 2} \mathbf{U}_{11}^{\mathrm{H}}$ into (19) and (20).

Achieving diagonal $\boldsymbol{\Gamma}_{0}$ in general requires a coupled matching network to "undo" the coupling created by the antenna. It is, however, common to assume that the coupled antenna can be represented using the diagonal elements of the full impedance matrix $\mathbf{Z}_{R}$, leading to a diagonal $\overline{\mathbf{S}}_{R}$ with elements $\bar{S}_{R, i i}=$ $\left(Z_{R, i i}-Z_{0}\right) /\left(Z_{R, i i}+Z_{0}\right)$. This value of $\overline{\mathbf{S}}_{R}$ is then used in place of $\mathbf{S}_{R}$ to specify the singular vectors and singular values as outlined above, leading to an uncoupled matching network. However, when analyzing the performance of such a match, the complete nondiagonal form of $\mathbf{S}_{R}$ must be used in the analysis equation (17).

\section{Diversity Gain}

Combining the network analysis of Section III with statistical incident field models allows quantification of the diversity gain achieved by the system. It is assumed that the antennas and incident waves have the same single polarization and that the incident field consists of an ensemble of plane waves with amplitudes drawn from a zero-mean complex Gaussian distribution. This assumption indicates that the received signal voltage will be a complex Gaussian random variable that can be completely specified in terms of the covariance $\mathbf{R}_{L}=$ $\mathrm{E}\left\{\mathbf{v}_{L} \mathbf{v}_{L}^{\mathrm{H}}\right\}$.

\section{A. Received Voltage Covariance}

Using the independence of the signal and noise waves, the covariance matrix $\mathbf{R}_{L}$ is given as

$$
\mathbf{R}_{L}=\mathbf{Q}\left[\mathbf{G R}_{S} \mathbf{G}^{\mathrm{H}}+\mathbf{R}_{\eta}\right] \mathbf{Q}^{\mathrm{H}}
$$

where $\mathbf{R}_{S}=\mathrm{E}\left\{\mathbf{b}_{S} \mathbf{b}_{S}^{\mathrm{H}}\right\}$ is the covariance of $\mathbf{b}_{S}$. The noise covariance is given as

$$
\begin{aligned}
\mathbf{R}_{\eta} & =\mathrm{E}\left\{\left(\boldsymbol{\Gamma}_{0} \mathbf{b}_{\eta}-\mathbf{a}_{\eta}\right)\left(\boldsymbol{\Gamma}_{0} \mathbf{b}_{\eta}-\mathbf{a}_{\eta}\right)^{\mathrm{H}}\right\} \\
& =k_{\mathrm{B}} B \underbrace{\left(T_{\alpha} \mathbf{I}+T_{\beta} \boldsymbol{\Gamma}_{0} \boldsymbol{\Gamma}_{0}^{\mathrm{H}}-T_{\gamma} \boldsymbol{\Gamma}_{0}-T_{\gamma}^{*} \boldsymbol{\Gamma}_{0}^{\mathrm{H}}\right)}_{T_{\alpha} \mathbf{R}_{\eta \circ}}
\end{aligned}
$$

where the results in (7) have been used. This expression explicitly shows how the value of $\boldsymbol{\Gamma}_{0}$ impacts the noise level (and, therefore, the SNR) at the receiver output.

It is assumed that the incident plane waves are uniformly distributed in the arrival angle within the horizontal plane and that their complex amplitudes are independent of the arrival angle and have variance $E^{2}$. Then, using (11) under the assumption of antennas and fields sharing the same polarization, the covariance of $\mathbf{b}_{S}$ may be written as

$$
\mathbf{R}_{S}=\frac{\left|c_{1}\right|^{2} E^{2}}{2 \pi Z_{0}} \int_{0}^{2 \pi} \mathbf{F}^{\prime T}(\phi) \mathbf{F}^{* *}(\phi) \mathrm{d} \phi .
$$

Using (25), it is interesting to explore the signal covariance at the matching network output ports, or $\mathbf{R}_{0}=\mathbf{G R}_{S} \mathbf{G}^{\mathrm{H}}$. First, using power conservation considerations, it is possible to show that $\mathbf{R}_{S}=c_{S}\left(\mathbf{I}-\mathbf{S}_{R} \mathbf{S}_{R}^{\mathrm{H}}\right)$, with $c_{S}$ a constant. Then, if $\boldsymbol{\Gamma}_{0}$ and, therefore, $\mathbf{U}_{0}$ are diagonal, use of the results in Section III-C shows that $\mathbf{R}_{0}$ is diagonal. Furthermore, if $\boldsymbol{\Lambda}_{R}=\lambda_{R} \mathbf{I}$ and $\boldsymbol{\Lambda}_{0}=\lambda_{0} \mathbf{I}$, then $\mathbf{R}_{0}=\lambda_{R 0} \mathbf{I}$. Effectively, this means that the coupled antenna and matching network combine the element patterns into array patterns that are orthogonal with equal (spatially averaged) gains, which also suggests that the coupled system maintains unit radiation efficiency, as demonstrated in [20]. Because this combining is performed before the amplifier noise is injected, the coupled system can achieve higher diversity than a system with uncoupled antennas.

\section{B. Equivalent Diversity Branches}

When the signal and noise waveforms received on each diversity branch are uncorrelated with the waveforms on the other branches, the statistics of the diversity-combined SNR level assuming maximal ratio or selection combining can be computed in closed form [1], [10]. However, in general, the covariance $\mathbf{R}_{L}$ computed in Section IV-A is not diagonal, indicating nonzero correlation. The received voltage $\mathbf{v}_{L}$ will therefore be transformed to diagonalize $\mathbf{R}_{L}$.

The first step in this transformation is to apply a prewhitening filter to decorrelate the received noise. To accomplish this, the eigenvalue decomposition (EVD) $\mathbf{R}_{\eta o}=\boldsymbol{\xi}_{\eta} \boldsymbol{\Lambda}_{\eta} \boldsymbol{\xi}_{\eta}^{\mathrm{H}}$ is computed, and $\boldsymbol{\zeta}=\mathbf{Q} \boldsymbol{\xi}_{\eta} \boldsymbol{\Lambda}_{\eta}^{1 / 2}$ is defined. $\mathbf{y}=\boldsymbol{\zeta}^{-1} \mathbf{v}_{L}$ is then defined, and its covariance is

$$
\begin{aligned}
\mathbf{R}_{y} & =\boldsymbol{\zeta}^{-1} \mathbf{R}_{L} \boldsymbol{\zeta}^{-H} \\
& =\underbrace{\boldsymbol{\Lambda}_{\eta}^{-\frac{1}{2}} \boldsymbol{\xi}_{\eta}^{\mathrm{H}} \mathbf{G} \mathbf{R}_{S} \mathbf{G}^{\mathrm{H}} \boldsymbol{\xi}_{\eta} \boldsymbol{\Lambda}_{\eta}^{-\frac{1}{2}}}_{\mathbf{R}_{S o}}+k_{\mathrm{B}} B T_{\alpha} \mathbf{I}
\end{aligned}
$$

which is seen to have uncorrelated noise. 
If the EVD of $\mathbf{R}_{S o}=\boldsymbol{\xi}_{S} \boldsymbol{\Lambda}_{S} \boldsymbol{\xi}_{S}^{\mathrm{H}}$ is computed next and $\mathbf{z}=$ $\boldsymbol{\xi}_{S}^{\mathrm{H}} \mathbf{y}$ is defined, the covariance of $\mathbf{z}$ can be computed as

$$
\begin{aligned}
\mathbf{R}_{z} & =\boldsymbol{\xi}_{S}^{\mathrm{H}} \mathbf{R}_{S o} \boldsymbol{\xi}_{S}+k_{\mathrm{B}} B T_{\alpha} \boldsymbol{\xi}_{S}^{\mathrm{H}} \boldsymbol{\xi}_{S} \\
& =\boldsymbol{\Lambda}_{S}+k_{\mathrm{B}} B T_{\alpha} \mathbf{I}
\end{aligned}
$$

where the unitary nature of $\boldsymbol{\xi}_{S}$ has been used. Therefore, $\mathbf{z}$ represents the output voltage vector of equivalent diversity branches with uncorrelated signals and noise. The elements of the diagonal matrix $\boldsymbol{\Lambda}_{S}$ represent the received signal power on each equivalent branch, while all branches have a noise power of $k_{\mathrm{B}} B T_{\alpha}$. The SNR for the $i$ th independent branch is therefore $\mathrm{SNR}_{i}=\Lambda_{S, i i} / k_{\mathrm{B}} B T_{\alpha}$. It is also noted that because $\mathbf{Q}$ multiplies both the signal and the noise, it does not appear in this diagonalized covariance representation and, therefore, will not impact the diversity performance.

\section{Effective Diversity Order}

The ultimate goal is to quantify the diversity performance of coupled antennas relative to the performance for uncoupled elements. The effective diversity order is an effective measure of overall performance since it includes both correlation and branch SNR in a single quantitative metric [10]. To compute this quantity for the coupled antennas, the cumulative distribution function (cdf) of the SNR for the diversity-combined signal is constructed. As previously mentioned, this cdf can be represented in closed form using the equivalent branch SNR values as computed in Section IV-B.

Now, a value pair (SNR, probability) on this cdf curve is selected. In this study, the SNR value corresponding to the $1 \%$ probability level is chosen, which means that the achieved SNR is lower than this value only $1 \%$ of the time. The number of uncoupled antennas that it would require to achieve this same SNR at the $1 \%$ point is then computed, assuming the signals on these antennas are uncorrelated. Since cdf curves can only be computed for integer numbers of independent signals, linear interpolation between the $1 \%$ points on these curves is used to find the fractional number of elements that would be required to achieve the given performance. This number represents the system diversity order.

To make this comparison meaningful, the branch SNR assumed for the uncorrelated reference signals must be consistent with the SNR levels for the coupled antenna system. To achieve this, the input impedance and radiation pattern for an isolated antenna are computed using the same electromagnetic technique as used for characterizing the coupled antennas. Using the framework in Section IV-A, the scalar variance $R_{S}$ (the matrix $\mathbf{F}^{\prime}$ becomes a simple column vector) for this single antenna in the environment of interest is computed. A matching network corresponding to $\boldsymbol{\Gamma}_{0}=\mathbf{0}$ is used for simplicity, yielding an average SNR for the single branch of $R_{S} / k_{\mathrm{B}} B T_{\alpha}$. The reference cdf curves for the independent branch signals are then constructed based on this branch SNR.

\section{Computational Examples}

\section{A. Antenna Electromagnetic Characterization}

To demonstrate application of the analysis framework developed in this paper and to illustrate the impact of antenna/ amplifier matching on the diversity performance of mutually coupled antennas, an array consisting of two coupled dipoles is explored. While closed-form expressions for coupled dipole impedance matrices exist (for reasonable antenna spacings), expressions for the patterns do not, motivating the use of fullwave electromagnetic solutions that can accurately characterize closely spaced elements. The finite-difference time-domain (FDTD) method [5], [21] was chosen for this computation. In this analysis, the $z$-oriented half-wave (total length) dipoles with a wire radius of $0.01 \lambda$ and separated by a distance $d$ are located at the center of the computational domain. Because narrowband systems are being considered here, single-frequency antenna excitation is used. The FDTD grid uses 80 cells per wavelength in the $z$ direction and 200 cells per wavelength in the $x$ and $y$ directions to adequately model the azimuthal current variations for close antenna spacings. A buffer region of a quarter wavelength is placed between the antennas and the terminating eight-cell perfectly matched layer (PML) absorbing boundary condition $(\mathrm{ABC})$.

Based upon the formulation in Section IV-A, pattern computations are performed when one antenna is excited, while the second is terminated in an open circuit. The antenna S-parameter matrix $\mathbf{S}_{R}$ is computed with the antennas terminated in $Z_{0}$. The same tool is used to characterize a single but otherwise identical dipole for use in the diversity order computation. The incident field consists of plane waves with uniformly distributed arrival angles such that the covariance $\mathbf{R}_{S}$ can be computed from (25).

The transistor used for the amplifier in this work is a bipolar junction transistor (BJT) taken from an application note [22]. At a collector-emitter bias voltage of $10 \mathrm{~V}$, collector current of $4 \mathrm{~mA}$, frequency of $4 \mathrm{GHz}$, and reference impedance of $Z_{0}=$ $50 \Omega$, the S-parameters and noise parameters are given as

$$
\begin{aligned}
\mathbf{S}_{11} & =0.552 \angle 169^{\circ} & & \mathbf{S}_{12}=0.049 \angle 23^{\circ} \\
\mathbf{S}_{21} & =1.681 \angle 26^{\circ} & & \mathbf{S}_{22}=0.839 \angle-67^{\circ} \\
F_{\min } & =2.5 \mathrm{~dB} & & \Gamma_{\mathrm{opt}}=0.475 \angle 166^{\circ} \\
R_{n} & =3.5 \Omega & &
\end{aligned}
$$

where $F_{\min }, \Gamma_{\mathrm{opt}}$, and $R_{n}$ are the device minimum noise figure, optimal source termination for noise figure, and effective noise resistance, respectively. These parameters are converted to the effective noise temperatures $T_{\alpha}, T_{\beta}$, and $T_{\gamma}$ using algebraic relations [19].

\section{B. Diversity Order Results}

In the examples, matching networks designed to achieve optimal amplifier noise figure ("NF"), optimal power gain, and $\boldsymbol{\Gamma}_{0}=\mathbf{0}$ are used. Matching network synthesis is based on the full antenna coupling matrix $\mathbf{S}_{R}$ as well as the diagonal coupling matrix $\overline{\mathbf{S}}_{R}$, as discussed in Section III-C. The abbreviation 


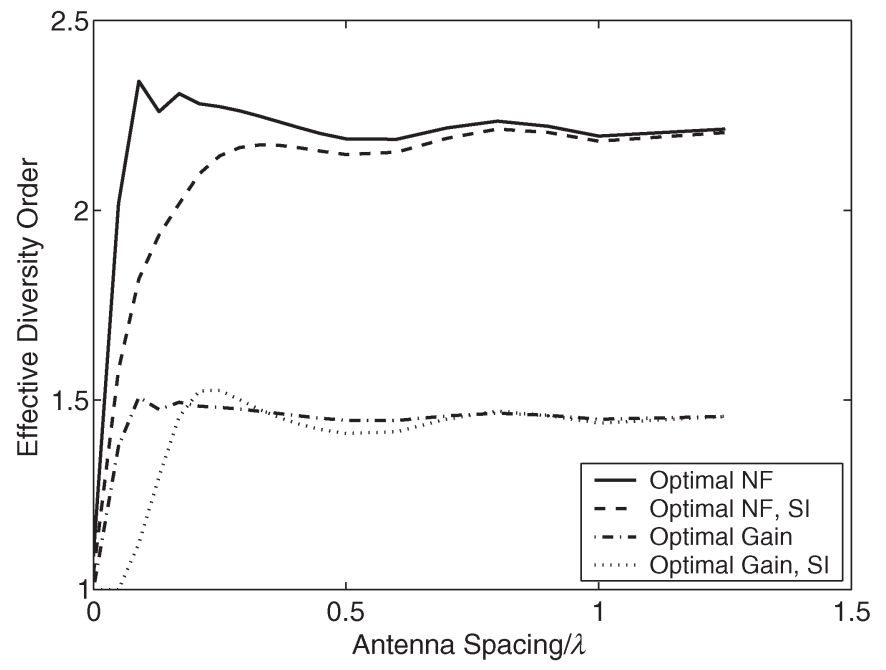

Fig. 3. Effective diversity order versus dipole spacing for matching networks that achieve optimal noise figure or optimal power gain for the amplifier. Curves are for optimal matching or for a matching network realized, assuming the antenna impedance matrix is diagonal ( $\mathrm{SI}=$ self-impedance).

"SI" (for self-impedance match) will be used in the plot legends to designate this latter case.

Fig. 3 plots the effective diversity order as a function of dipole spacing for matching networks achieving optimal noise figure and power gain. Several observations regarding these results deserve attention. First, for very close antenna spacings, the two antennas behave largely as a single element, resulting in a diversity order near unity. This low diversity order increases rapidly with separation, however, and for certain moderate spacings, it can actually exceed the diversity order achieved for large element separation. This peak in the diversity order stems from the pattern orthogonality created by the coupled antennas and matching network, as discussed in Section IV-A, which leads to higher diversity than can be achieved with the uncoupled dipoles with the same spacing used as a reference in the diversity order computation. Such a phenomenon was not observed in prior results [11], [12] since such coupled matching networks were not examined. The results of Fig. 3 also show that matching to the self-impedance creates relatively little degradation in performance, particularly for element spacings larger than about $\lambda / 4$.

The final and perhaps most revealing finding from the results in Fig. 3 is that while optimal power transfer is a typical design goal, it is dramatically suboptimal in terms of diversity performance. This is an intuitive result, since matching for maximum power transfer neglects the impact of the match on amplifier noise figure, which directly controls the received $\mathrm{SNR}$, which is the key parameter in determining the overall communication performance. This superiority of matching for minimum noise figure is therefore general for any receiving system equipped with practical noisy amplifiers. Prior studies in which the accurate amplifier noise model is not included [12], [14], [16] are not capable of predicting this behavior since in such a case, the match only impacts the power and not the noise, leading to the conclusion that matching for maximum power transfer is optimal.

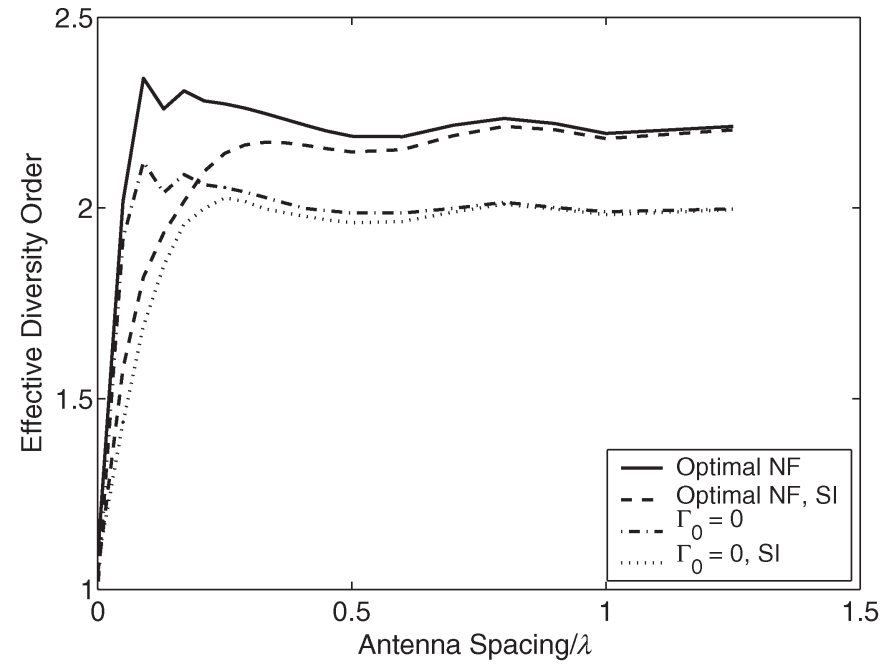

Fig. 4. Effective diversity order versus dipole spacing for matching networks that achieve optimal noise figure for the amplifier or zero output reflection. Curves are for optimal matching or for a matching network realized, assuming the antenna impedance matrix is diagonal ( $\mathrm{SI}=$ self-impedance).

It is important to emphasize that the uncoupled reference dipoles used in the diversity order computation are terminated in the suboptimal match achieving $\boldsymbol{\Gamma}_{0}=\mathbf{0}$, which explains why the diversity order is generally larger than 2 when a match for optimal noise figure is implemented. If, however, the matching network for the coupled antennas also achieves $\boldsymbol{\Gamma}_{0}=\mathbf{0}$, then the diversity order is expected to approach a value of 2 for large spacings where coupling is weak. This intuition is confirmed in Fig. 4, which shows the diversity order for this case compared to the results for the optimal noise figure. However, this result also shows an increase in diversity order above 2 for certain spacings, which is a phenomenon that stems from the increased power collection capability of the coupled dipoles relative to the uncoupled ones, as discussed in conjunction with the results of Fig. 3. It is also noted that while $\boldsymbol{\Gamma}_{0}=\mathbf{0}$ is suboptimal both in terms of noise figure and power transfer, it performs much better for this device than the optimal power gain match considered in Fig. 3.

\section{CONCLUSION}

This paper has presented a new analysis of receiving diversity systems consisting of mutually coupled antennas, matching networks, and independent front-end amplifiers for each antenna. The analysis uses a network theory to formulate the voltage at the amplifier outputs for an incident electric field and provides a mechanism for computing the covariance of these voltages. The noise model for the amplifiers includes both forward and reverse traveling noise waves, which is a detail not previously considered and which notably complicates the formulation. Using the SVD to characterize the S-parameter matrix of the matching network allowed specification of these S-parameters for matching networks to achieve the desired design goals. Examples involving electromagnetic characterization of two coupled dipoles coupled with the network analysis revealed that on the receiver, matching networks designed to achieve 
a minimum noise figure for the amplifiers provide superior performance to those designed to achieve optimal signal power transfer through the network. This conclusion is general to any practical small signal receive amplifier configuration with a nonzero noise figure, since ultimately, SNR is the key parameter in determining the diversity performance. It is important to point out, however, that this finding is not generally applicable for diversity transmitters where transmitted power, as opposed to a noise figure, is the dominant consideration.

\section{APPENDIX}

Lossless matching networks are characterized by unitary S-matrices such that $\mathbf{S}_{M}^{\mathrm{H}} \mathbf{S}_{M}=\mathbf{I}$. Using the representation in (2) and substituting the SVD of the subblocks $\mathbf{S}_{i j}=$ $\mathbf{U}_{i j} \Lambda_{i j}^{1 / 2} \mathbf{V}_{i j}^{\mathrm{H}}$ into the lossless constraint yields the relations

$$
\mathbf{V}_{i j} \boldsymbol{\Theta}_{i j}=\mathbf{V}_{j j} \quad \boldsymbol{\Lambda}_{i j}=\mathbf{I}-\boldsymbol{\Lambda}_{j j}, \quad i \neq j
$$

where $\boldsymbol{\Theta}_{i j}$ is a diagonal matrix with unit-magnitude entries. This operation also produces the condition

$$
\begin{aligned}
& \boldsymbol{\Lambda}_{11}^{\frac{1}{2}} \mathbf{U}_{11}^{\mathrm{H}} \mathbf{U}_{12}\left(\mathbf{I}-\boldsymbol{\Lambda}_{22}\right)^{\frac{1}{2}} \boldsymbol{\Theta}_{12} \\
&=-\Theta_{21}^{\mathrm{H}}\left(\mathbf{I}-\boldsymbol{\Lambda}_{11}\right)^{\frac{1}{2}} \mathbf{U}_{21}^{\mathrm{H}} \mathbf{U}_{22} \boldsymbol{\Lambda}_{22}^{\frac{1}{2}} .
\end{aligned}
$$

There is an entire family of matching networks that satisfy these conditions. Since finding one lossless matching network that achieves specified design goals is the main emphasis here, the singular vectors/values can be further specified. In this spirit, $\mathbf{U}_{12}=\mathbf{U}_{11}$ and $\mathbf{U}_{21}=\mathbf{U}_{22}$ are chosen. Then, according to (30), $\boldsymbol{\Theta}_{21}=-\boldsymbol{\Theta}_{12}^{\mathrm{H}}=\boldsymbol{\Theta}$ and $\boldsymbol{\Lambda}_{22}=\boldsymbol{\Lambda}_{11}$ are obtained. The subblocks of $\mathbf{S}_{M}$ can then be expressed as in (3).

\section{REFERENCES}

[1] W. C. Jakes, Microwave Mobile Communications. New York: IEEE Press, 1993.

[2] C. A. Balanis, Antenna Theory: Analysis and Design. New York: Wiley, 1997.

[3] K. Boyle, "Radiation patterns and correlation of closely spaced linear antennas," IEEE Trans. Antennas Propag., vol. 50, no. 8, pp. 1162-1165, Aug. 2002.

[4] S. C. K. Ko and R. D. Murch, "Compact integrated diversity antenna for wireless communications," IEEE Trans. Antennas Propag., vol. 49, no. 6, pp. 954-960, Jun. 2001.

[5] M. A. Jensen and Y. Rahmat-Samii, "Performance analysis of antennas for hand-held transceivers using FDTD," IEEE Trans. Antennas Propag., vol. 42, no. 8, pp. 1106-1113, Aug. 1994.

[6] M. C. Leifer, "Signal correlations in coupled cell and MIMO antennas," in Proc. IEEE Antennas Propagation Society Int. Symp., San Antonio, TX, Jun. 16-21, 2002, vol. 3, pp. 194-197.

[7] J. Luo, J. R. Zeidler, and S. McLaughlin, "Performance analysis of compact antenna arrays with MRC in correlated Nakagami fading channels," IEEE Trans. Veh. Technol., vol. 50, no. 1, pp. 267-277, Jan. 2001.

[8] P. S. H. Leather and D. Parsons, "Antenna diversity for UHF handportable radio," Electron. Lett., vol. 39, no. 13, pp. 946-948, Jun. 2003.

[9] T. Svantesson and A. Ranheim, "Mutual coupling effects on the capacity of multielement antenna systems," in Proc. IEEE Int. Conf. Acoustics, Speech, Signal Processing, Salt Lake City, UT, May 7-11, 2001, vol. 4, pp. 2485-2488.

[10] O. Nørklit, P. D. Teal, and R. G. Vaughan, "Measurement and evaluation of multi-antenna handsets in indoor mobile communication," IEEE Trans. Antennas Propag., vol. 49, no. 3, pp. 429-437, Mar. 2001.
[11] R. G. Vaughan and J. B. Andersen, "Antenna diversity in mobile communications," IEEE Trans. Veh. Technol., vol. VT-36, no. 4, pp. 147-172, Nov. 1987.

[12] W. C. Y. Lee, "Mutual coupling effect on maximum-ratio diversity combiners and application to mobile radio," IEEE Trans. Commun., vol. COM-18, no. 6, pp. 779-791, Dec. 1970.

[13] J. W. Wallace and M. A. Jensen, "Intrinsic capacity of the MIMO wireless channel," in Proc. IEEE Antennas Propagation Soc. Int. Symp., San Antonio, TX, Jun. 16-21, 2002, vol. 3, pp. 198-201.

[14] R. G. Vaughan and N. L. Scott, "Closely spaced monopoles for mobile communications," Radio Sci., vol. 28, no. 6, pp. 1259-1266, Nov.-Dec. 1993.

[15] C. Waldschmidt, J. V. Hagen, and W. Wiesbeck, "Influence and modeling of mutual coupling in MIMO and diversity systems," in Proc. IEEE Antennas Propagation Soc. Int. Symp., San Antonio, TX, Jun. 16-21, 2002, vol. 3, pp. 190-193.

[16] J. W. Wallace and M. A. Jensen, "Termination-dependent diversity performance of coupled antennas: Network theory analysis," IEEE Trans. Antennas Propag., vol. 52, no. 1, pp. 98-105, Jan. 2004.

[17] D. M. Pozar, Microwave Engineering. New York: Wiley, 1998.

[18] G. Gonzalez, Microwave Transistor Amplifiers. Englewood Cliffs, NJ: Prentice-Hall, 1997.

[19] J. Engberg and T. Larsen, Noise Theory of Linear and Nonlinear Circuits. New York: Wiley, 1995.

[20] S. Stein, "On cross coupling in multiple-beam antennas," IRE Trans. Antennas Propag., vol. AP-10, no. 5, pp. 548-557, Sep. 1962.

[21] A. Taflove and S. C. Hagness, Computational Electrodynamics: The Finite-Difference Time-Domain Method, 2nd ed. Boston, MA: Artech House, 2000.

[22] Hewlett-Packard, "A low noise $4 \mathrm{GHz}$ transistor amplifier using the HXTR-6101 silicon bipolar transistor," in Hewlett-Packard Application Note 967, May 1975.

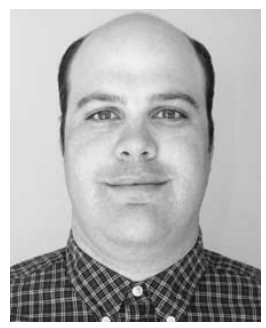

Matthew L. Morris received the B.S. degree in physics from Brigham Young University (BYU), Provo, UT, in 2000, where he is currently working toward a Ph.D. degree in electrical engineering.

His research interests include multiple-input multiple-output communication with an emphasis on channel capacity.

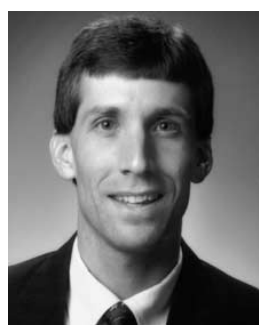

Michael A. Jensen (S'93-M'95-SM'01) received the B.S. (summa cum laude) and M.S. degrees in electrical engineering from Brigham Young University (BYU), Provo, UT, in 1990 and 1991, respectively, and the Ph.D. degree in electrical engineering at the University of California, Los Angeles (UCLA), in 1994.

From 1989 to 1991 , he was a graduate research assistant in the Lasers and Optics Laboratory at BYU. From 1991 to 1994 , he was a graduate student researcher in the Antenna Laboratory at UCLA. Since 1994, he has been at the Electrical and Computer Engineering Department at BYU, where he is currently an Associate Professor. His main research interests include antennas and propagation for personal communications, microwave circuit design, radar remote sensing, numerical electromagnetics, and optical fiber communications.

Dr. Jensen currently serves on the Joint Meetings Committee for the IEEE Antennas and Propagation Society and has served as the Technical Program Chair for the 2000 IEEE International Symposium on Antennas and Propagation and Symposium Vice Chair for the 2003 IEEE Topical Conference on Wireless Communications Technology. He received the H. A. Wheeler Paper Award for a paper in the IEEE TRANSACTIONS ON ANTENNAS AND PROPAGATION in 2002 and the Best Paper Award at the 1994 IEEE International Symposium on Antennas and Propagation. He is a member of Eta Kappa Nu and Tau Beta Pi. In 1990, he received a National Science Foundation Graduate Fellowship. 